\title{
Mediating pathways from central obesity to childhood asthma: a population-based longitudinal study
}

\author{
An-Hsuan Chih ${ }^{1,2}$, Yang-Ching Chen ${ }^{3,4}$, Yu-Kang Tu1, Kuo-Chin Huang ${ }^{5}$, \\ Tai-Yuan Chiu ${ }^{5}$ and Yungling Leo Lee ${ }^{1,6}$
}

Affiliations: ${ }^{1}$ Institute of Epidemiology and Preventive Medicine, College of Public Health, National Taiwan University, Taipei, Taiwan. ${ }^{2}$ Health Centre, Office of Student Affairs, National Taiwan University, Taipei, Taiwan. ${ }^{3}$ Taipei City Hospital, ZhongXing Branch, Taipei, Taiwan. ${ }^{4}$ School of Nutrition and Health Sciences, Taipei Medical University, Taipei, Taiwan. ${ }^{5}$ Dept of Family Medicine, National Taiwan University Hospital, Taipei, Taiwan. Institute of Biomedical Sciences, Academia Sinica, Taipei, Taiwan.

Correspondence: Yungling L. Lee, Institute of Epidemiology and Preventive Medicine, College of Public Health, National Taiwan University, 17 Xu-Zhou Road, 516R, ZhongZheng District, Taipei 100, Taiwan.

E-mail: leoleedntu.edu.tw

ABSTRACT The mediating pathways linking obesity and asthma are largely unknown. We aimed to investigate the mediating pathways and to search for the most prominent pathological mechanism between central obesity and childhood asthma.

In the Taiwan Children Health Study, we collected data on an open cohort of children aged 9-13 years. Children's respiratory outcomes, atopic conditions, obesity measures and pulmonary function were surveyed annually between 2010 and 2012. Exhaled nitric oxide fraction concentrations were recorded in 2012. Generalised estimating equations and general linear models were used to examine the associations between central obesity, possible mediators and asthma. Structural equation models were applied to investigate the pathways that mediate the link between central obesity and asthma.

Central obesity (waist-to-hip ratio) most accurately predicted childhood asthma. In the active asthma model, the percentage of mediation was $28.6 \%$ for pulmonary function, $18.1 \%$ for atopy and $5.7 \%$ for airway inflammation. The percentage of mediation for pulmonary function was $40.2 \%$ in the lifetime wheeze model. Pulmonary function was responsible for the greatest percentage of mediation among the three mediators in both models.

Decline in pulmonary function is the most important pathway in central obesity related asthma. Pulmonary function screening should be applied to obese children for asthma risk prediction.

@ERSpublications

Decline in pulmonary function is the most prominent pathological pathway from central obesity to childhood asthma http://ow.ly/4mJomZ

This article has supplementary material available from erj.ersjournals.com

Received: June 182015 | Accepted after revision: April 012016 | First published online: May 122016

Support statement: This study was supported by grants \#98-2314-B-002-138-MY3 and \#96-2314-B-006-053 from the Taiwan National Science Council.

Conflict of interest: None declared.

Copyright OERS 2016 


\section{Introduction}

Obesity and asthma are important health issues for children worldwide. Two recent meta-analyses of prospective cohorts reported that children with high body mass index (BMI) exhibit an increased risk of physician-diagnosed asthma and incident asthma [1,2]. Beside BMI, other obesity indicators may provide additional information [3]. MUSAAD et al. [4] and our group [5] both found that central obesity, as measured by waist circumference or waist-to-hip ratio, was the most useful obesity indicator of childhood asthma. Although a certain "obese asthmatic" phenotype may exist [6,7], little is known about the pathological mechanism underlying the relationship between central obesity and asthma.

Several pathways might explain the pathophysiology underlying obesity and asthma. Atopy is related to immunological mechanisms of asthma, and commonly occurs in asthmatic children [8]. Two studies have demonstrated that overweight children have an increased risk of atopy and asthma [9, 10], but other studies have reported conflicting results [11, 12]. Recently, ForNo et al. [3] demonstrated that atopy is a significant mediator in the obesity-asthma link. Exhaled nitric oxide fraction ( $F e N O)$, which can provide a quantitative measure of airway inflammation, was associated with allergic asthma in children [13]. A previous study has claimed that overweight children with asthma and high FeNO levels have worse asthma control than normal-weight children with asthma and high FeNO levels [14]. In addition, children with severe asthma exhibit features of both atopy and increased FeNO [15]. Therefore, we believe that atopy and airway inflammation are mediators in the pathogenesis from central obesity to asthma.

Obesity decreases lung capacity [16] and may subsequently cause asthma. A study revealed that abdominal adiposity predicted pulmonary function better than BMI, suggesting the importance of central obesity to limited lung expansion [17]. Our previous study also demonstrated that central obesity-related decline in pulmonary function (measured by forced expiratory volume in $1 \mathrm{~s}$ (FEV1)/forced vital capacity (FVC)) led to childhood asthma, but we did not investigate other mechanisms underlying the central obesity-asthma link [5]. Additionally, the relative effects of various mediators in the link remain to be clarified.

The Taiwan Children Health Study (TCHS) is a nationwide population-based cohort covering diverse regions of Taiwan that provides a good opportunity to test our hypotheses in a longitudinal design. We continued our previous study [5] and hypothesised that the link between central obesity and childhood asthma could be mediated by atopy, airway inflammation and poor pulmonary function. The mediating effect of each pathway was also evaluated to search for the most prominent pathological mechanism underlying this link.

\section{Methods}

\section{Data collection}

The TCHS is a multidisciplinary longitudinal nationwide study, focusing on the respiratory health and associated factors of schoolchildren. The details of TCHS have been described in our previous study [5]. It comprises two cohorts, and our study used data from the second cohort with an open-cohort design. Every year from 2010 to 2012, the same questionnaire was distributed to parents to assess the children's respiratory health, atopic conditions and associated confounders. Data from the parent questionnaire and the children's obesity measures and pulmonary function tests were collected annually from April to June between 2010 and 2012. FeNO was recorded in the 2012 survey. Written informed consent was obtained from the parents of all the child participants. The study protocol was approved by the institutional review board of the National Taiwan University Hospital (Taipei, Taiwan) (approval number: 200902042R) and followed the principles outlined in the Declaration of Helsinki [18].

\section{Measurement}

\section{Outcomes}

We used the parent questionnaire to define the respiratory outcomes of active asthma and lifetime wheeze. The following two questions were used for active asthma: "Has a doctor ever diagnosed your child as having asthma?" and "In the past 12 months, did your child experience difficulty breathing, or did you observe any wheezing or whistling from his or her chest?" If parents answered "yes" to both questions, the child was classified as an active asthma case. The question for lifetime wheeze was "Did your child ever experience difficulty breathing, or did you observe any wheezing or whistling from his or her chest?" The questionnaire responses of the parents were used to categorise the respiratory outcomes of the child participants in the baseline and follow-up surveys.

\section{Obesity}

Body weight was measured with the participants dressed in light clothing without shoes. The electronic scale used was a Body Composition Analyzer (IOI 353; Jawon Medical, Gyeongsan-si, Korea; accuracy: $0.1 \mathrm{~kg}$ ). Body height was determined to the nearest $1 \mathrm{~mm}$ using a wall-mounted stadiometer (BW-120; 
Nagata, Yung Kang City, Taiwan) at each school. Waist and hip circumferences were measured to the nearest $1 \mathrm{~mm}$ using a flexible tape. Skinfold thickness was measured in duplicate to the closest $0.5 \mathrm{~mm}$ using Lange calipers (Beta Technology, Santa Cruz, CA, USA) at the bilateral triceps and gastrocnemius. The same investigator took all measurements based on our previous standardised procedures [5]. The average skinfolds of the bilateral triceps and gastrocnemius were added to obtain the skinfold sums, and the body fat percentage was calculated according to formulas from a previously published study [19]:

$$
\begin{aligned}
& \text { Body fat percentage in males }=0.735 \times(\text { triceps }+ \text { gastrocnemius })+1.0 \\
& \text { Body fat percentage in females }=0.610 \times(\text { triceps }+ \text { gastrocnemius })+5.1
\end{aligned}
$$

\section{Atopy}

Three atopic diseases, allergic rhinitis, atopic dermatitis and atopic conjunctivitis, were used to represent atopic conditions. The parent-reported questionnaire was adopted to define the lifetime occurrence of the three diseases diagnosed by physicians. The responses of the parents on the baseline and follow-up questionnaires were used to categorise the atopic conditions of the child participants.

\section{Airway inflammation}

We used FeNO measurements as a surrogate marker of eosinophilic airway inflammation. A NIOX MINO airway inflammation monitor (Aerocrine AB, Solna, Sweden) was used in the 2012 survey. The standardised procedures for measuring exhaled nitric oxide were based on the recommendations of the American Thoracic Society and the European Respiratory Society [20]. Child participants were asked to inhale air free of nitric oxide to total lung capacity and then exhale over a period of $10 \mathrm{~s}$ at a flow rate of $50 \mathrm{~mL} \cdot \mathrm{s}^{-1}$. The concentration of nitric oxide in the exhaled mixed gas was displayed on the screen after analysis.

\section{Pulmonary function tests}

Pulmonary function tests were performed according to our previously standardised protocol [21]. FEV1 and FVC data were obtained using spirometers. The FEV1/FVC ratio was calculated.

\section{Data analysis}

We first classified the different obesity measures into three categories $(<25 \%, \geqslant 25-<85 \%$ and $\geqslant 85 \%)$ by age- and sex-specific percentiles using the distribution of our study population. To examine the relationships between obesity measures and the respiratory outcomes (active asthma and lifetime wheeze) throughout the study period, we employed generalised estimating equations (GEE). The GEE analysis, which uses a class of estimating equations, is an extension of generalised linear models (GLMs) for repeated observations [22]. The correlation matrix adopted in the GEE analysis was a working first-order autoregressive correlation. A variety of confounders were entered into the GEE analysis, including age, sex, parental education level, household income, family history of atopy, whether the child had been breastfed and whether the child was exposed to maternal smoke in utero. Subjects with missing covariate information were included in the model using missing indicators [23]. The level of statistical significance was set at $5 \%$ based on a two-sided estimate.

We conducted a mediation analysis to assess whether indirect effects via the mediators explained part of the association between central obesity (waist-to-hip ratio) and the respiratory outcomes. A structural equation model (SEM) analysis was performed because it was a good way to test and control multiple pathways simultaneously [24]. In addition, the SEM eliminated estimation bias that occurred when multiple intercorrelated mediators were tested individually in a simple mediation model [25]. Latent variables were used in the SEM to reduce the measurement errors arising in repeated measurements from the three surveys. We assumed that the pathological pathways from central obesity to the respiratory outcomes were mediated by atopy, airway inflammation and pulmonary function. Data on the waist-to-hip ratios from the three surveys were used to construct the latent variable "central obesity". A linear combination was used for the respiratory outcomes from the 2010-2012 surveys. For each atopic disease, we used linear combination to combine data from all three surveys, and then the three atopic diseases were combined to construct the latent variable "atopy". Data on the FeNO measurements were used in the mediation analysis to represent "airway inflammation". Data on the FEV1/FVC values for the 3 years studied were used to construct the latent variable "pulmonary function". FEV1/FVC was chosen to represent pulmonary function because it was associated with adiposity indicators in children with asthma and increased FeNO levels in a large nationwide survey [14]. To establish a model, the standardised pathway coefficients between central obesity and the mediators as well as between the mediators and the respiratory outcomes must both reach statistical significance. The number of bootstrap draws was set as 
500. A full information maximum likelihood estimation was used for missing data in the SEM. The percentage of mediation of each mediator was calculated as the estimate of the indirect effect divided by the estimate of the total effect of the model [3]. The standardised pathway coefficients as well as the standardised total, direct and indirect effects were considered statistically significant if $<5 \%$. Mplus software (version 7; Muthén \& Muthén, Los Angeles, CA, USA) was used to perform the SEM analysis.

To test whether the introduction of the confounders changed the significance of the mediators between central obesity and the respiratory outcomes, we undertook the GEE and GLM analyses. The GEE analysis was used for the mediators of atopy and pulmonary function, whereas the GLM analysis was used for the mediator of airway inflammation. Atopy in the GEE analysis was defined as whether parents reported any one of the three atopic diseases in the child participants in each survey. The correlation matrix adopted in the GEE analysis, the confounders adjusted and the processing of missing data were the same as described in the previous paragraphs. SAS software version 9.2 (SAS Institute, Cary, NC, USA) was used to perform the GEE and GLM analyses. The level of statistical significance was set at 5\% based on a two-sided estimation.

\section{Results}

The study population consisted of 2777 fourth-grade schoolchildren in 2010, 2170 fifth-grade children in 2011, and 2311 sixth-grade children in 2012. The prevalence of active asthma declined from $4.8 \%$ in the 2010 survey to $3.5 \%$ in the 2012 survey (table 1). During the 3 -year study period, the percentage of child participants who had a lifetime wheeze ranged from $14.2 \%$ to $21.7 \%$. In the 2010 survey, $40.3 \%$ of the children had at least one of the three atopic diseases. The mean values for FEV $1 / \mathrm{FVC}$ were $90.7 \%$ and $91.6 \%$ in the 2010 and 2012 surveys, respectively.

Table 2 displays the associations between the obesity measures and respiratory outcomes using GEE. BMI percentile, body fat percentage and waist-to-hip ratio all revealed a significant dose-response relationship

\begin{tabular}{|c|c|c|c|}
\hline & 2010 & 2011 & 2012 \\
\hline Subjects ${ }^{\#}$ & 2777 & 2170 & 2311 \\
\hline Males & $1411(50.8)$ & $1077(49.6)$ & $1162(50.3)$ \\
\hline Age years & $9.7 \pm 0.5$ & $10.6 \pm 0.50$ & $11.7 \pm 0.5$ \\
\hline Parental education years & $12.9 \pm 2.2$ & $12.9 \pm 2.2$ & $12.9 \pm 2.3$ \\
\hline \multicolumn{4}{|l|}{ Household income per month } \\
\hline$<50000$ TWD & $1145(41.2)$ & 926 (42.7) & $940(40.7)$ \\
\hline 50000-100000 TWD & $1023(36.8)$ & $795(36.6)$ & $878(38.0)$ \\
\hline$>100000$ TWD & $509(18.3)$ & $373(17.2)$ & $418(18.1)$ \\
\hline In utero smoke exposure & $86(3.1)$ & 70 (3.2) & $70(3.0)$ \\
\hline Breastfeeding & $1407(50.7)$ & $1092(50.3)$ & $1188(51.4)$ \\
\hline Family history of atopy & $955(34.4)$ & $750(34.6)$ & 803 (34.8) \\
\hline \multicolumn{4}{|l|}{ Obesity measures } \\
\hline Body mass index $\mathrm{kg} \cdot \mathrm{m}^{-2}$ & $18.6 \pm 3.6$ & $19.2 \pm 3.5$ & $20.3 \pm 3.9$ \\
\hline Body fat (skinfolds) \% & $23.2 \pm 8.0$ & $27.7 \pm 8.2$ & $25.4 \pm 7.4$ \\
\hline Waist-to-hip ratio \% & $81.5 \pm 5.9$ & $81.7 \pm 5.8$ & $77.3 \pm 6.7$ \\
\hline \multicolumn{4}{|l|}{ Respiratory outcomes } \\
\hline Active asthma & $134(4.8)$ & $105(4.8)$ & 80 (3.5) \\
\hline Lifetime wheeze & 394 (14.2) & $471(21.7)$ & $487(21.1)$ \\
\hline \multicolumn{4}{|l|}{ Atopy ${ }^{\text {I }}$} \\
\hline AR & $891(32.1)$ & 886 (40.8) & $867(37.5)$ \\
\hline$A D$ & 331 (11.9) & $318(14.7)$ & 374 (16.2) \\
\hline $\mathrm{AC}$ & $356(12.8)$ & $379(17.5)$ & 375 (16.2) \\
\hline$A R$ or $A D$ or $A C$ & $1120(40.3)$ & 1049 (48.3) & $1045(45.2)$ \\
\hline \multicolumn{4}{|l|}{ Pulmonary function } \\
\hline $\mathrm{FEV}_{1} / \mathrm{FVC} \%$ & $90.7 \pm 6.7$ & $91.8 \pm 6.1$ & $91.6 \pm 6.5$ \\
\hline \multicolumn{4}{|l|}{ Airway inflammation } \\
\hline FeNO ppb & & & $11.5 \pm 17.1$ \\
\hline
\end{tabular}

Data are presented as $\mathrm{n}, \mathrm{n}(\%)$ or mean \pm SD. TWD: Taiwan dollars; AR: allergic rhinitis; AD: atopic dermatitis; AC: acute conjunctivitis; FEV1: forced expiratory volume in $1 \mathrm{~s}$; FVC: forced vital capacity; FeNO: exhaled nitric oxide fraction. " : missing data; " 


\begin{tabular}{|c|c|c|}
\hline & Active asthma & Lifetime wheeze \\
\hline \multicolumn{3}{|l|}{ BMI percentiles } \\
\hline$<25 \%$ & 1 & 1 \\
\hline $25 \%-<85 \%$ & $1.33(0.98-1.81)$ & $1.19(0.99-1.42)$ \\
\hline$\geqslant 85 \%$ & $1.88(1.24-2.85)$ & $1.60(1.25-2.05)$ \\
\hline $\mathrm{p}$-value for trend & 0.003 & $<0.001$ \\
\hline \multicolumn{3}{|c|}{ Body fat percentage (skinfold) } \\
\hline$<25 \%$ & 1 & 1 \\
\hline $25-<85 \%$ & $0.95(0.69-1.30)$ & $1.02(0.85-1.22)$ \\
\hline$\geqslant 85 \%$ & $1.70(1.16-2.51)$ & $1.44(1.12-1.85)$ \\
\hline $\mathrm{p}$-value for trend & 0.03 & 0.01 \\
\hline \multicolumn{3}{|l|}{ Waist-to-hip ratio } \\
\hline$<25 \%$ & 1 & 1 \\
\hline $25-<85 \%$ & 1.70 (1.24-2.33) & $1.21(1.01-1.44)$ \\
\hline$\geqslant 85 \%$ & $2.07(1.36-3.15)$ & $1.50(1.18-1.90)$ \\
\hline $\mathrm{p}$-value for trend & $<0.001$ & 0.001 \\
\hline
\end{tabular}

Data are presented as OR $(95 \% \mathrm{Cl})$, unless otherwise stated. All models were adjusted for age, sex, parental education level, household income, in utero smoke exposure, breastfeeding and family history of atopy. $\mathrm{p}$-values for trend were calculated using a generalised estimating equation model. BMI: body mass index.

in the prediction of active asthma and lifetime wheeze. Of the three obesity measures, waist-to-hip ratio was the best predictor of active asthma. For child participants with waist-to-hip ratio $\geqslant 85$ th percentile, the odds ratios (95\% CI) for active asthma and lifetime wheeze were 2.07 (1.36-3.15) and 1.50 (1.18-1.90), respectively. All the obesity indicators exhibited better associations with active asthma compared with lifetime wheeze; recently measured obesity indicators should more accurately reflect the present status of asthma rather than the past occurrence of wheeze.

We used the SEM to investigate the mediating pathways underlying the mechanisms from central obesity (waist-to-hip ratio) to the respiratory outcomes. Table 3 presents the standardised path coefficients for each pathway when the mediators were introduced. Positive correlations were found between atopy and airway inflammation as well as between airway inflammation and pulmonary function. The direct links from central obesity to the respiratory outcomes in both models were positive but were not significant ( $p$-values 0.06 and 0.16 for the model of active asthma and lifetime wheeze, respectively). Figure 1 is a diagram of the SEM results outlined in table 3.

Table 4 presents the standardised total, direct and indirect effects of pathways from central obesity to the respiratory outcomes in the SEM. The total effects and indirect effects via different mediators were all significant in both models (except for borderline significance via airway inflammation in the model of lifetime wheeze, $\mathrm{p}$-value $=0.07$ ). In the active asthma model, the percentage of mediation was $28.6 \%$ for pulmonary function, $18.1 \%$ for atopy and $5.7 \%$ for airway inflammation. The indirect effect via pulmonary function was $40.2 \%$ in the lifetime wheeze model. Pulmonary function was responsible for the greatest percentage of mediation among the three mediators in both models.

The associations between central obesity and the mediators using the GEE and GLM analyses are presented in table 5. Central obesity showed a significant dose-response relationship with the three mediators. For child participants with waist-to-hip ratio $\geqslant 85$ th percentile, the FeNO was 2.58 (95\% CI 0.11-5.05) ppb higher and the FEV1/FVC was 1.41 (95\% CI $0.87-1.95) \%$ lower than the reference group. The associations between the mediators and the respiratory outcomes using the GEE and GLM analyses are presented in table 6. All three mediators significantly predicted active asthma and lifetime wheeze. The interquartile range was $13 \mathrm{ppb}$ for $\mathrm{FeNO}$ and $8.69 \%$ for FEV1/FVC. For child participants with atopy, the odds ratio (95\% CI) for active asthma was 3.77 (2.76-5.15). An interquartile increase in FeNO was related to a 1.26 (1.13-1.42)-fold greater risk of children exhibiting active asthma. With respect to pulmonary function, an interquartile increase in FEV1/FVC was associated with an 18 (3-31)\% lower risk of active asthma.

For the GEE analysis using a working unstructured correlation matrix, refer to online supplementary tables S1, S2 and S3. The results were similar to those reported in tables 2, 5 and 6 . For the GEE analysis using data of child participants who attended all three surveys without any missing values, refer to online supplementary tables S4, S5 and S6. The associations between variables did not differ much whether we used the complete dataset $(n=1864)$ or the data of the original open cohort with missing indicators. 
TABLE 3 Standardised path coefficients for pathways from central obesity to respiratory outcomes by mediators in a structural equation model

\begin{tabular}{|c|c|c|c|c|}
\hline & \multicolumn{2}{|c|}{ Active asthma } & \multicolumn{2}{|c|}{ Lifetime wheeze } \\
\hline & Coefficient & p-value & Coefficient & p-value \\
\hline \multicolumn{5}{|l|}{ Pathway } \\
\hline Positive link from central obesity to atopy & 0.09 & 0.01 & 0.09 & 0.01 \\
\hline Positive link from central obesity to airway inflammation & 0.06 & 0.01 & 0.06 & 0.01 \\
\hline Negative link from central obesity to pulmonary function & -0.33 & $<0.001$ & -0.33 & $<0.001$ \\
\hline Negative link from pulmonary function to respiratory outcomes & -0.09 & 0.001 & -0.14 & $<0.001$ \\
\hline Positive link from atopy to airway inflammation & 0.24 & $<0.001$ & 0.24 & $<0.001$ \\
\hline Negative link from airway inflammation to pulmonary function & -0.06 & 0.04 & -0.05 & 0.05 \\
\hline \multicolumn{5}{|l|}{ Model fitness } \\
\hline Chi-squared test of model fit $p$-value & \multicolumn{2}{|c|}{$<0.001$} & \multicolumn{2}{|c|}{$<0.001$} \\
\hline $\mathrm{CFI}$ & \multicolumn{2}{|c|}{0.99} & \multicolumn{2}{|c|}{0.99} \\
\hline
\end{tabular}

CFI: comparative fit index; TLI: Tucker-Lewis index; RMSEA: root mean square error of approximation.

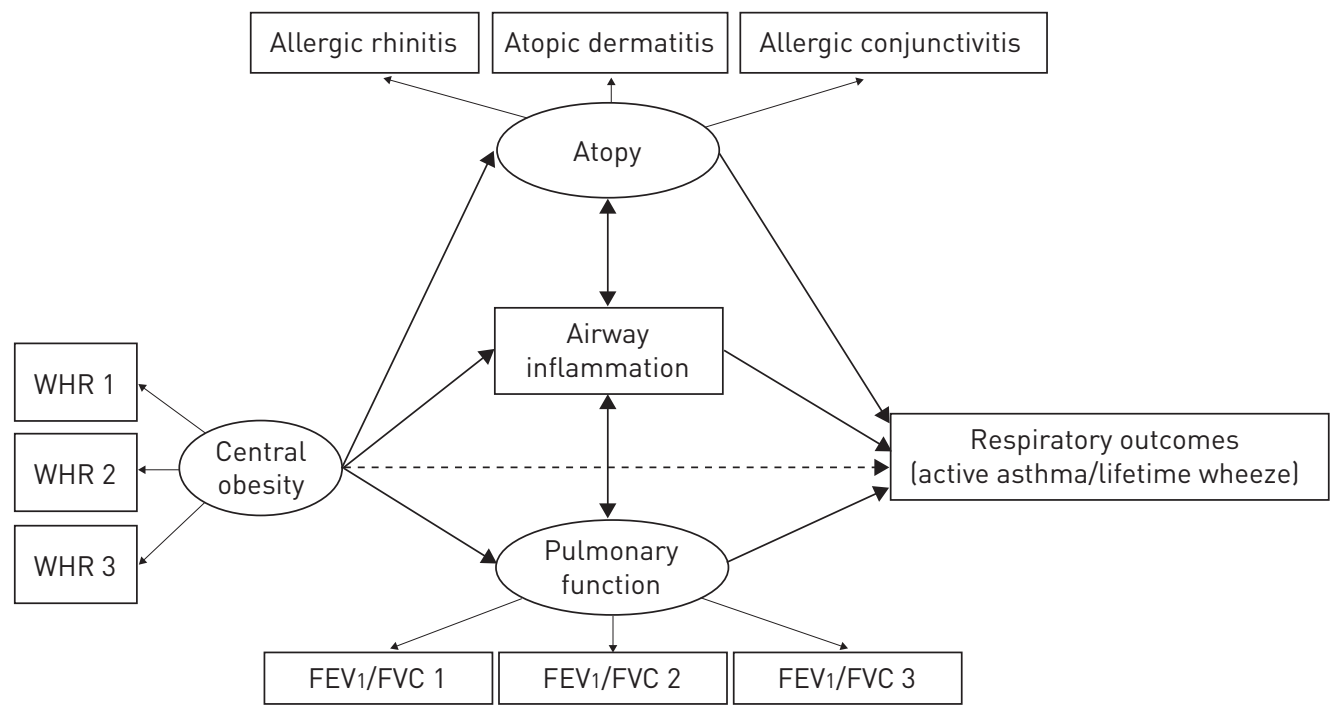

FIGURE 1 Structural equation model with pathways from central obesity to respiratory outcomes by different mediators. Latent variables are depicted as ovals; rectangles represent indicators and other measured variables. Significant relationships are represented by large arrows; insignificant relationships are represented by dashed arrows. The directions of the arrows distinguish between predictors and outcomes. WHR: waist-to-hip ratio; FEV1: forced expiratory volume in $1 \mathrm{~s}$; FVC: forced vital capacity.

\section{Discussion}

To our knowledge, this is the first study to discuss the mediating effects of pathways from central obesity to asthma. Our findings demonstrated that atopy, airway inflammation and poor pulmonary function are significant mediators of the link between central obesity and childhood asthma. Among these mediators, poor pulmonary function was the most prominent pathological mechanism contributing to this link. Because improving pulmonary function by physical training is effective and costs lower than pharmacological treatment to reverse atopy and airway inflammation, our findings have important implications for the respiratory health of children.

In paediatric studies, higher BMI values were found to be associated with nonatopic asthma rather than atopic asthma [11]. However, ForNo et al. [3] considered atopy to be a significant mediator of the obesityasthma link. In our study, we investigated three atopic diseases to better characterise "atopy", and obese 
TABLE 4 Standardised effects for pathways from central obesity to respiratory outcomes by mediators in a structural equation model

\begin{tabular}{|c|c|c|c|c|}
\hline & \multicolumn{2}{|c|}{ Active asthma } & \multicolumn{2}{|c|}{ Lifetime wheeze } \\
\hline & Estimate & p-value & Estimate & p-value \\
\hline Total effect & 0.105 (100.0) & $<0.001$ & $0.112(100.0)$ & $<0.001$ \\
\hline Direct effect ${ }^{\#}$ & $0.049(46.7)$ & 0.06 & $0.036(32.1)$ & 0.16 \\
\hline Total indirect effect ${ }^{\Uparrow}$ & $0.055(52.4)$ & $<0.001$ & $0.076(67.9)$ & $<0.001$ \\
\hline Indirect effect ${ }^{\text {ๆ }}$ via atopy & $0.019(18.1)$ & 0.01 & $0.027(24.1)$ & 0.01 \\
\hline Indirect effect ${ }^{\text {ๆ }}$ via airway inflammation & $0.006(5.7)$ & 0.04 & $0.004(3.6)$ & 0.07 \\
\hline Indirect effect ${ }^{\text {ๆ }}$ via pulmonary function & $0.030(28.6)$ & 0.01 & $0.045(40.2)$ & $<0.001$ \\
\hline
\end{tabular}

Data are presented as coefficients of pathways (\% mediated), unless otherwise stated. ${ }^{\#}$ : direct relationship between central obesity and respiratory outcomes; ": mediation of mediators in the relationship between central obesity and respiratory outcomes.

children reported more atopic conditions, similar to the results found in other studies [26]. Our findings were consistent with the ForNo et al. study and demonstrate that atopy significantly mediates the effect of central obesity on asthma. Central obesity might yield more information than BMI with respect to atopic conditions. Discrepancies among the available studies with respect to obesity, atopy and asthma might be explained by diverse genetic and environmental factors [3].

FeNO is highly correlated with eosinophilic rather than neutrophilic airway inflammation [27]. Eosinophilic airway inflammation is important in the obesity-asthma link because submucosal eosinophil counts have been correlated with BMI in patients with asthma [28]. A monoclonal antibody against human interleukin (IL)-5, which had a key role in the promotion of eosinophil survival, was associated with significantly fewer severe exacerbations of asthma [29]. Treatment strategies aimed at reducing sputum eosinophil counts also decreased asthma admissions and exacerbations [30]. A large cross-sectional study in children in USA demonstrated that obesity was associated with poor asthma control in children with high FeNO levels [14]. In our study, using waist-to-hip ratio, airway inflammation was found to be a significant mediator between central obesity and asthma, but the mediating effect was small. This might be because obesity-related systemic inflammation would not directly enhance airway inflammation [31, 32]. It was noteworthy that airway inflammation correlated with atopy and pulmonary function in our study (table 3 and fig. 1), and these findings were compatible with other reports in children [33,34]. We believe that atopy, airway inflammation and pulmonary function may act synergistically to cause asthma symptoms in children with central obesity.

It is known that obesity reduces pulmonary function in subjects with or without asthma. In female asthma patients, the effect of dynamic hyperinflation was greater in those with a higher BMI [35]. As SHORE and FREDBERG [36] noted, obesity influenced pulmonary function by mechanisms of compromised bronchodilatation, accelerated airway remodelling and impaired lung growth. The role of lung function that mediated the link between central obesity and asthma was also confirmed in our previous study [5].

TABLE 5 Associations between central obesity and atopy, airway inflammation and pulmonary function using a generalised estimating equation model

$\frac{\text { Atopy }^{\#}}{\text { OR }(95 \% \mathrm{Cl})} \quad \frac{\text { Airway inflammation (FeNo) ppb }}{\beta(95 \% \mathrm{Cl})} \quad \frac{\text { Pulmonary function (FEV } 1 / \mathrm{FVC}) \%}{\beta(95 \% \mathrm{Cl})}$

\begin{tabular}{lccc}
\hline $\begin{array}{l}\text { Central obesity waist-to-hip ratio } \\
<25 \%\end{array}$ & Ref. & Ref. & Ref. \\
$25-<85 \%$ & $1.11(1.04-1.19)$ & $2.28(0.62-3.93)$ & $-0.40(-0.77--0.03)$ \\
$\geqslant 85 \%$ & $1.11(1.00-1.22)$ & $2.58(0.11-5.05)$ & $-1.41(-1.95--0.87)$ \\
p-value for trend & 0.01 & 0.01 & $<0.001$
\end{tabular}

All models were adjusted for age, sex, parental education level, household income, in utero smoke exposure, breastfeeding and family history of atopy. p-values for trend were calculated using a generalised estimating equation model (atopy and pulmonary function) or general linear model (airway inflammation). FeNO: exhaled nitric oxide fraction; FEV1: forced expiratory volume in $1 \mathrm{~s}$; FVC: forced vital capacity; Ref: reference. "\#: parental report of any one of allergic rhinitis, atopic dermatitis or allergic conjunctivitis in the child participants in each survey. 
TABLE 6 Associations between atopy, airway inflammation, pulmonary function and respiratory outcomes using a generalised estimating equation model

\begin{tabular}{ccc} 
& Active asthma & Lifetime wheeze \\
\hline Atopy $^{\#}$ & $3.77(2.76-5.15)$ & $2.30(1.91-2.76)$ \\
p-value & $<0.001$ & $<0.001$ \\
Airway inflammation (FeNo per 13 ppb change) & $1.26(1.13-1.42)$ & $1.20(1.10-1.30)$ \\
p-value & $<0.001$ & $<0.001$ \\
Pulmonary function (FEV1/FVC per 8.69\% change) & $0.82(0.69-0.97)$ & $0.86(0.78-0.94)$ \\
p-value & 0.02 & 0.002 \\
\end{tabular}

Data are presented as OR $(95 \% \mathrm{Cl})$, unless otherwise stated. All models were adjusted for age, sex, parental education level, household income, in utero smoke exposure, breastfeeding and family history of atopy. $p$-values were calculated using a generalised estimating equation model latopy and pulmonary function) or general linear model (airway inflammation). FeNO: exhaled nitric oxide fraction; FEV1: forced expiratory volume in $1 \mathrm{~s}$; FVC: forced vital capacity. ${ }^{\#}$ : parental report of any one of allergic rhinitis, atopic dermatitis or allergic conjunctivitis in the child participants in each survey.

We interchanged the respiratory outcomes with pulmonary function in the SEM analysis to test whether asthma mediated the effect of obesity on pulmonary function. However, the fitness information of the new models was worse than that of our original models (online supplementary table S7). Therefore, we believe that asthma is less likely to be a mediator between central obesity and pulmonary function. In addition, our study was the first to demonstrate the mediating effect of pulmonary function on the link between central obesity and asthma was greater than that of atopy and airway inflammation. Our analysis also revealed that pulmonary function had a greater effect on lifetime wheeze than on active asthma. As previous longitudinal studies have proven, this was probably because children with wheeze or asthma in early life exhibited progressive pulmonary function deficits in later life [37, 38]. In our study, the participants who did not have lifetime wheeze in the 2010 survey but answered "yes" to lifetime wheeze in the 2012 survey showed worse pulmonary function when compared with those who had never wheezed (online supplementary table S8). Therefore, the greater effect of pulmonary function on lifetime wheeze might result from a pulmonary function deficit of extended duration. Our findings suggest that obese children should be started on physical training as early as possible to mitigate obesity and the deterioration of pulmonary function.

Regarding the possible molecular mechanisms linking central obesity and asthma, several studies have shown promising results. Leptin is a proinflammatory adipokine produced by adipose tissue, the level of which increases in obesity. A study by GULER et al. [39] confirmed that serum leptin concentrations exhibited positive correlation with IgE levels in children with asthma. In a mouse model of asthma, accumulation of visceral body fat has been revealed to lower allergic sensitisation by production of IgE and IL-5. Obesity also promoted allergic airway inflammation by eosinophilia. These findings explain the key role obesity plays in allergy-related cases of childhood asthma [40]. Chitinase-3-like 1 (CHI3L1) is a chitinase-like protein known to exhibit positive correlation with truncal obesity in humans. In obese subjects with asthma, serum levels of CHI3L1 were found to be associated with poor pulmonary function [41].

The major strengths of our study are the repeated measurements and the large sample size of a community cohort. Most of the data were collected annually for 3 years. We used lifetime wheeze as one of the main outcomes because "wheeze at least once" was a more common condition than an asthma diagnosis in children and thus identified more patients with impaired pulmonary function [42]. Moreover, we obtained similar results in two different statistical models with two distinct respiratory outcomes, which made our interpretation more reliable.

Our study was limited by the questionnaire-derived survey of child atopic conditions and respiratory outcomes; however, the parents, who were mature enough to provide the most accurate responses answered the questionnaire. We also used questions regarding physician-diagnosed atopic diseases and objective measurements to avoid possible classification bias. Data on FeNO were limited to the 2012 survey. Nevertheless, the effects of airway inflammation were significant in our analyses and cannot be ignored. Another limitation was that we did not assess puberty in our study. However, we used age as a proxy for puberty staging in the GEE models, and the results were significant for each pathway.

In conclusion, decline in pulmonary function, in comparison with atopy and airway inflammation, is the most prominent pathological mechanism from central obesity to childhood asthma. In view of the great burden of obesity and asthma, our findings have important implications for disease-prevention policies. 
Pulmonary function screening should be applied to obese children to predict asthma risk. Improving pulmonary function is the best strategy to prevent asthma related to central obesity.

\section{Acknowledgements}

We would like to thank the children and parents who participated in this study. We would also like to thank the Taiwan Children Health Study team members (College of Public Health, National Taiwan University, Taipei, Taiwan) for their assistance in the collection of cohort data.

\section{References}

1 Egan KB, Ettinger AS, Bracken MB. Childhood body mass index and subsequent physician-diagnosed asthma: a systematic review and meta-analysis of prospective cohort studies. BMC Pediatr 2013; 13: 121.

2 Chen YC, Dong GH, Lin KC, et al. Gender difference of childhood overweight and obesity in predicting the risk of incident asthma: a systematic review and meta-analysis. Obes Rev 2013; 14: 222-231.

3 Forno E, Acosta-Pérez E, Brehm JM, et al. Obesity and adiposity indicators, asthma, and atopy in Puerto Rican children. J Allergy Clin Immunol 2014; 133: 1308-1314.

4 Musaad SM, Patterson T, Ericksen M, et al. Comparison of anthropometric measures of obesity in childhood allergic asthma: central obesity is most relevant. J Allergy Clin Immunol 2009; 123: 1321-1327.

5 Chen YC, Tu YK, Huang KC, et al. Pathway from central obesity to childhood asthma. Physical fitness and sedentary time are leading factors. Am J Respir Crit Care Med 2014; 189: 1194-1203.

6 Sutherland ER, Goleva E, King TS, et al. Cluster analysis of obesity and asthma phenotypes. PLoS One 2012; 7: e36631.

7 Jensen ME, Collins CE, Gibson PG, et al. The obesity phenotype in children with asthma. Paediatr Respir Rev 2011; 12: 152-159.

8 Forno E, Cloutier MM, Datta S, et al. Mouse allergen, lung function, and atopy in Puerto Rican children. PLoS One 2012; 7: e40383.

9 Murray CS, Canoy D, Buchan I, et al. Body mass index in young children and allergic disease: gender differences in a longitudinal study. Clin Exp Allergy 2011; 41: 78-85.

10 Cibella F, Cuttitta G, La Grutta S, et al. A cross-sectional study assessing the relationship between BMI, asthma, atopy, and eNO among schoolchildren. Ann Allergy Asthma Immunol 2011; 107: 330-336.

11 Visness CM, London SJ, Daniels JL, et al. Association of childhood obesity with atopic and nonatopic asthma: results from the National Health and Nutrition Examination Survey 1999-2006. J Asthma 2010; 47: 822-829.

12 Kattan M, Kumar R, Bloomberg GR, et al. Asthma control, adiposity, and adipokines among inner-city adolescents. J Allergy Clin Immunol 2010; 125: 584-592.

13 Scott M, Raza A, Karmaus W, et al. Influence of atopy and asthma on exhaled nitric oxide in an unselected birth cohort study. Thorax 2010; 65: 258-262.

14 Han YY, Forno E, Celedón JC. Adiposity, fractional exhaled nitric oxide, and asthma in U.S. children Am J Respir Crit Care Med 2014; 190: 32-39.

15 Fitzpatrick AM, Gaston BM, Erzurum SC, et al. Features of severe asthma in school-age children: atopy and increased exhaled nitric oxide. J Allergy Clin Immunol 2006; 118: 1218-1225.

16 Beuther DA, Sutherland ER. Obesity and pulmonary function testing. J Allergy Clin Immunol 2005; 115: $1100-1101$.

17 Ochs-Balcom HM, Grant BJ, Muti P, et al. Pulmonary function and abdominal adiposity in the general population. Chest 2006; 129: 853-862.

18 World Medical Association declaration of Helsinki. Recommendations guiding physicians in biomedical research involving human subjects. JAMA 1997; 277: 925-926.

19 Slaughter MH, Lohman TG, Boileau RA, et al. Skinfold equations for estimation of body fatness in children and youth. Hum Biol 1988; 60: 709-723.

20 American Thoracic Society; European Respiratory Society. ATS/ERS recommendations for standardized procedures for the online and offline measurement of exhaled lower respiratory nitric oxide and nasal nitric oxide, 2005. Am J Respir Crit Care Med 2005; 171: 912-930.

21 Lee YL, Hwang BF, Chen YA, et al. Pulmonary function and incident bronchitis and asthma in children: a community-based prospective cohort study. PLoS One 2012; 7: e32477.

22 Liang KY, Zeger SL. Longitudinal data analysis using generalized linear models. Biometrika 1986; 73: 13-22.

23 Huberman M, Langholz B. Application of the missing-indicator method in matched case-control studies with incomplete data. Am J Epidemiol 1999; 150: 1340-1345.

24 Wood RE, Goodman JS, Beckmann N, et al. Mediation testing in management research: a review and proposals. Organ Res Methods 2008; 11: 270-295.

25 Preacher KJ, Hayes AF. Asymptotic and resampling strategies for assessing and comparing indirect effects in multiple mediator models. Behav Res Methods 2008; 40: 879-891.

26 Silverberg JI, Kleiman E, Lev-Tov H, et al. Association between obesity and atopic dermatitis in childhood: a case-control study. J Allergy Clin Immunol 2011; 127: 1180-1186.

27 Dweik RA, Boggs PB, Erzurum SC, et al. An official ATS clinical practice guideline: interpretation of exhaled nitric oxide levels $\left(F_{\mathrm{E}_{\mathrm{NO}}}\right)$ for clinical applications. Am J Respir Crit Care Med 2011; 184: 602-615.

28 Desai D, Newby C, Symon FA, et al. Elevated sputum interleukin-5 and submucosal eosinophilia in obese individuals with severe asthma. Am J Respir Crit Care Med 2013; 188: 657-663.

29 Haldar P, Brightling CE, Hargadon B, et al. Mepolizumab and exacerbations of refractory eosinophilic asthma. N Engl J Med 2009; 360: 973-984.

30 Green RH, Brightling CE, McKenna S, et al. Asthma exacerbations and sputum eosinophil counts: a randomised controlled trial. Lancet 2002; 360: 1715-1721.

31 Sideleva O, Suratt BT, Black KE, et al. Obesity and asthma: an inflammatory disease of adipose tissue not the airway. Am J Respir Crit Care Med 2012; 186: 598-605.

32 Sutherland TJ, Cowan JO, Young S, et al. The association between obesity and asthma: interactions between systemic and airway inflammation. Am J Respir Crit Care Med 2008; 178: 469-475. 
33 Gabriele C, Nieuwhof EM, Van Der Wiel EC, et al. Exhaled nitric oxide differentiates airway diseases in the first two years of life. Pediatr Res 2006; 60: 461-465.

34 Yao TC, Ou LS, Lee WI, et al. Exhaled nitric oxide discriminates children with and without allergic sensitization in a population-based study. Clin Exp Allergy 2011; 41: 556-564.

35 Sutherland TJ, Cowan JO, Taylor DR. Dynamic hyperinflation with bronchoconstriction: differences between obese and nonobese women with asthma. Am J Respir Crit Care Med 2008; 177: 970-975.

36 Shore SA, Fredberg JJ. Obesity, smooth muscle, and airway hyperresponsiveness. J Allergy Clin Immunol 2005; 115: 925-927.

37 Martinez FD, Wright AL, Taussig LM, et al. Asthma and wheezing in the first six years of life. N Engl J Med 1995; 332: $133-138$.

38 Bisgaard H, Jensen SM, Bønnelykke K. Interaction between asthma and lung function growth in early life. Am J Respir Crit Care Med 2012; 185: 1183-1189.

39 Guler N, Kirerleri E, Ones U, et al. Leptin: does it have any role in childhood asthma? J Allergy Clin Immunol 2004; 114: 254-259.

40 Dietze J, Böcking C, Heverhagen JT, et al. Obesity lowers the threshold of allergic sensitization and augments airway eosinophilia in a mouse model of asthma. Allergy 2012; 67: 1519-1529.

41 Ahangari F, Sood A, Ma B, et al. Chitinase 3-like-1 regulates both visceral fat accumulation and asthma-like Th2 inflammation. Am J Respir Crit Care Med 2015; 191: 746-757.

42 Depner M, Fuchs O, Genuneit J, et al. Clinical and epidemiologic phenotypes of childhood asthma. Am J Respir Crit Care Med 2014; 189: 129-138. 